\title{
Changes of frequency spectrum of the CSF pulse wave caused by supratentorial epidural brain compression
}

\author{
HIDEO TAKIZAWA, THEA GABRA-SANDERS, J DOUGLAS MILLER \\ From the Department of Surgical Neurology, University of Edinburgh, Scotland, UK
}

SUMMARY The frequency spectrum of the CSF pulse wave and amplitude transfer function from arterial to CSF pressure were estimated in the lateral ventricle and cisterna magna during the course of supratentorial epidural compression of brain in anaesthetised ventilated cats. Ventricular fluid pressure, the amplitude of spectral components of the CSF pulse and the amplitude of transfer function in the supratentorial compartment were increased in an exponential manner as epidural compression advanced. A transtentorial pressure gradient developed when epidural compression exceeded a critical level. Conduction of the CSF pulse through the tentorial hiatus decreased in an exponential manner when plotted against the transtentorial pressure gradient. Transtentorial conduction of the CSF pulse may serve as a sensitive indicator of the development of tentorial herniation.

Changes of the waveform of the CSF pulse associated with changes in intracranial pressure (ICP) are often noted during continuous monitoring. Several authors have proposed that waveform analysis of the CSF pulse may be a useful means to estimate intracranial pressure dynamics. ${ }^{1-5}$ Detailed study of the CSF pulse waveform under controlled experimental conditions, however, is still necessary before assessing the practical value of pressure waveform analysis in the management of patients with raised ICP. This study was planned to determine the behaviour of the CSF pulse wave when ICP was raised experimentally by epidural compression of the brain under controlled conditions.

\section{Materials and methods}

Twelve mongrel cats of both sexes, weighing between 4.2 and $4.8 \mathrm{~kg}$, were used for these experiments. Each animal was anaesthetized by ketamine $20 \mathrm{mg} / \mathrm{kg}$ IM after premedication by atropine $0.05 \mathrm{mg} / \mathrm{kg}$ subcutaneously. Tracheotomy and intubation were performed, then anaesthesia was maintained by mixed gases of $30 \% \mathrm{O}_{2}$ and $70 \% \mathrm{~N}_{2} \mathrm{O}$. The animal was paralysed by pancuronium bromide $0.05 \mathrm{mg} / \mathrm{kg}$ IV and respiration was maintained mechanically (Harvard respiratory Model 665 A made by Harvard Apparatus,

Address for reprint requests: Dr $\mathbf{H}$ Takizawa, Department of Surgery, Tokyo Rohsai Hospital, Ohmoni-minami 4-13-21, Ohtaku, Tokyo 143, Japan.

Received 18 October 1985 and in revised form 3 March 1986. Accepted 8 March 1986
South Natick, Massachusetts, USA). The left femoral vein and artery were cannulated for the drip infusion of Hartmann's solution and for monitoring of blood gases (blood gas analyser IL system 1302 made by Instrument Laboratory, Milano, Italy), respectively. A catheter was placed in the left subclavian artery for monitoring of systemic blood pressure.

The animal was fixed in a stereotaxic frame in the sphinx position. A midline incision was made in the suboccipital region and a 22-gauge spinal needle was placed in the cisterna magna through the foramen magnum. A small rubber balloon was placed in the epidural space of the right parietal region through a drill hole in the skull. Another spinal needle of the same gauge was inserted into the left lateral ventricle stereotaxically through a drill hole. The sites of insertion of spinal needles and epidural balloon were sealed by cyanoacrylate adhesive or dental acrylic.

The catheter placed in the subclavian artery and spinal needles in the CSF space were connected to pressure transducers (pressure transducers made by Bentley Laboratories, Irvine, California, USA) by tubes of high pressure type and of equal length for recording by a polygraph and a FM tape recorder (FM tape recorder Model MR-10 made by TEAC Corporation, Tokyo, Japan). All pressure transducers were calibrated by a water column and positioned halfway between the tip of dorsal spine and the sternum. Pulsatile components of arterial pressure and CSF pulse were selectively magnified by a four channel AC amplifier with preset gain from twice to 100 times, and recorded by a FM tape recorder.

After stabilisation of the animal, the CSF pulse and blood pressure were recorded under baseline conditions. Then the epidural balloon was inflated at the rate of $0.038 \mathrm{ml} / \mathrm{min}$ and all pressures were recorded. Sizes of both pupils were 


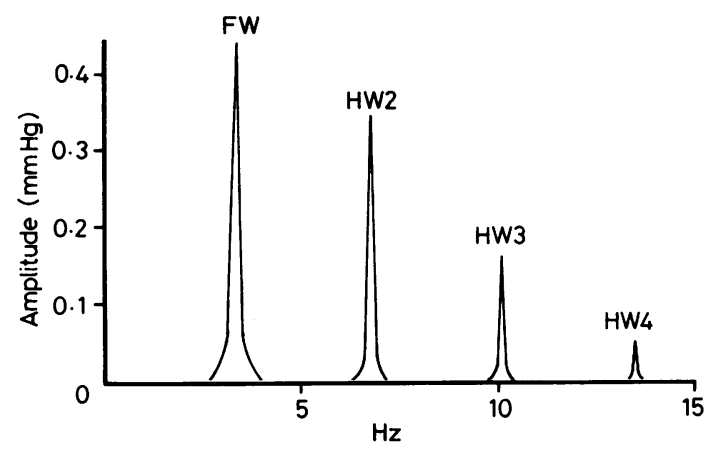

Fig 1 The basic pattern of the frequency spectrum of the CSF pulse wave. The spectrum contains four components derived from cardiac beats: $F W=$ fundamental wave, $H W 2$ $=2$ nd harmonic wave, $H W 3=3 r d$ harmonic wave, $H W 4=$ 4th harmonic wave.

observed intermittently. The experiment was terminated when the balloon was inflated to $2.0 \mathrm{ml}$.

We define a transtentorial pressure gradient as the difference between CSF pressures estimated in the lateral ventricle and the cisterna magna.

Spectral analysis of the collected arterial and CSF pressure data was performed after the end of the experiment. The signal from the FM tape recorder was sampled by a 12 bit analog-to-digital converter at a rate of $50 \mathrm{~Hz}$, and the frequency spectrum of each pressure waveform was calculated by a 16 bit personal computer using an algorithm for a 512 point Fast Fourier Transform. The range of frequency spectrum was between 0.6 and $18.1 \mathrm{~Hz}$ and the frequency resolution was $0.098 \mathrm{~Hz}$. In this study, we analysed only the spectral components derived from cardiac beats, ignoring the lower frequency component associated with respiration.

The frequency response of the pressure measuring system was tested and confirmed to be flat up to $20 \mathrm{~Hz}$.

Nomenclature of spectral components

An example of the spectrum of the CSF pulse wave is shown in fig 1. Four sharp peaks are present; this pattern of spectrum was basically the same for both blood pressure and the CSF pulse. The definition of abbreviations for spectral components are:

$\mathrm{FW}=$ fundamental wave (or first harmonic wave)

HW2 $=$ 2nd harmonic wave

HW3 = 3rd harmonic wave

HW4 = 4th harmonic wave

Amplitude transfer function

The amplitude transfer function from arterial blood pressure to CSF pulse wave was calculated by the following equation: ${ }^{1}$

$\operatorname{TFa}(\mathrm{x})=$

amplitude of spectral component (x) of CSF pulse amplitude of spectral component (x) of blood pressure

where $\operatorname{TFa}(x)=$ amplitude transfer function of spectral component $(x)$

$(x=F W$, HW2. HW3 or HW4)
Transtentorial conduction of CSF pulse

The conduction of each spectral component of the CSF pulse wave from supratentorial to infratentorial compartments through the tentorial hiatus was calculated by following equation:

$$
\operatorname{Cond}(\mathrm{x})=\frac{\operatorname{Amp}(\mathrm{x} / \mathrm{CM})}{\operatorname{Amp}(\mathrm{x} / \mathrm{LV})} \times 100 \%
$$

where Cond $(x)=$ conduction of spectral component $(x)$ of the CSF pulse wave

$\operatorname{Amp}(\mathrm{x} / \mathrm{CM})=$ amplitude of spectral component $(\mathrm{x})$

of the CSF pulse in the cisterna magna

$\operatorname{Amp}(x / L V)=$ amplitude of spectral component (x)

of the CSF pulse in the lateral ventricle

$\mathrm{x}=\mathrm{FW}, \mathrm{HW} 2, \mathrm{HW} 3$ or HW4

\section{Results}

Two cats were discarded from the series because the needle in the lateral ventricle became blocked at an early stage of epidural compression of the brain.

During the course of experiments, arterial blood gases were stabilised; $\mathrm{pH}, \mathrm{PO}_{2}$ and $\mathrm{pCO}_{2}$ were $7.39 \pm$ $0.04,183 \pm 26 \mathrm{~mm} \mathrm{Hg}$ and $28.5 \pm 2.4 \mathrm{~mm} \mathrm{Hg}$ (mean and SD), respectively.

There was no significant change of systemic arterial pressure, as shown in fig 2.

\section{(1) Changes of the intracranial pressure and transtentorial gradient of CSF pressure}

The CSF pressure in the lateral ventricle (VFP) was raised progressively by the inflation of an epidural balloon as shown in fig 3(a). In the beginning of the epidural compression, there was no difference between CSF pressures in the lateral ventricle and cisterna magna. A transtentorial pressure gradient appeared when the volume of the epidural balloon exceeded $0.38 \mathrm{ml}$ (fig 2 (b)). The volume of the bal-

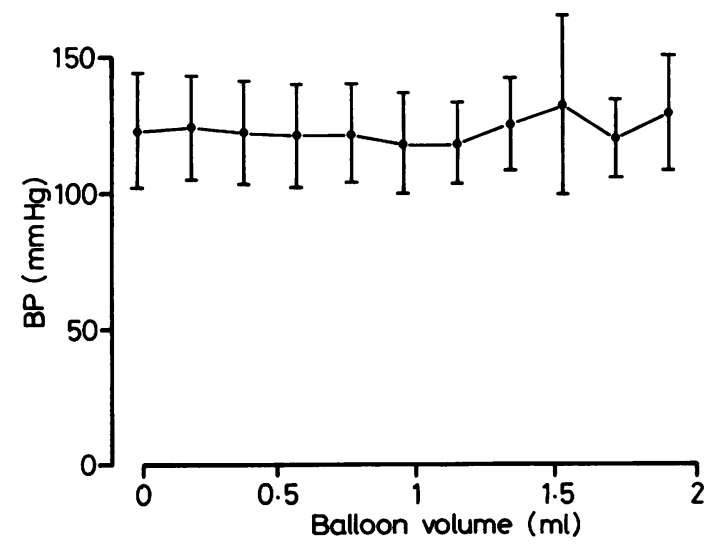

Fig 2 Changes of mean blood pressure in the course of epidural balloon inflation. Mean and SD are shown. 

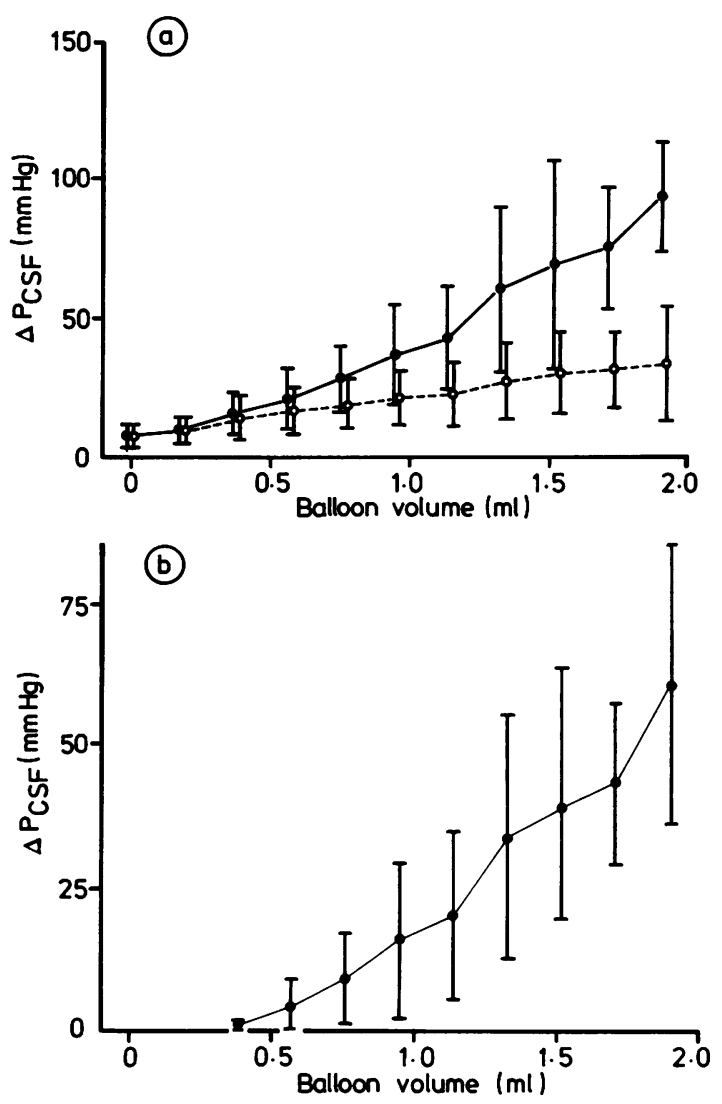

Fig 3 Changes of CSF pressure $\left(P_{\mathrm{CSF}}\right)$ in the lateral ventricle (closed circles) and cisterna magna (open circles) (b) development of the transtentorial pressure gradient $\left(\Delta P_{\mathrm{CSF}}\right)$ in the course of epidural balloon inflation. Mean and $S D$ are shown. $\Delta P_{\mathrm{CSF}}=$ ventricular $C S F$ pressure - cisterna magna CSF pressure.

loon, when ipsilateral dilatation of the pupil appeared, was $1.28 \pm 0.27 \mathrm{ml}$ (mean and SD) and the corresponding CSF pressure levels in the lateral ventricle and the transtentorial pressure gradient were $53.6 \pm 15.9$ and $28.3 \pm 7.8 \mathrm{~mm} \mathrm{Hg}$ respectively.

\section{(2) Changes of amplitude of spectral components of the CSF pulse wave}

The pattern of changes was basically the same for all four spectral components of the CSF pulse as shown in fig 4; (a) the amplitude values for each spectral component in the lateral ventricle and cisterna magna were almost identical in the early stage of the epidural balloon inflation, (b) the amplitudes of all spectral components of the CSF pulse in the lateral ventricle were increased progressively as the epidural com- pression progressed and (c) amplitudes of all components of the CSF pulse in the cisterna magna were, in contrast, increased only slightly.

(3) Changes of amplitude transfer function from blood pressure to the CSF pulse wave

The values of amplitude transfer function from blood pressure to the CSF pulse estimated in the lateral ventricle were increased progressively during epidural compression of the brain. In contrast, those estimated in the cisterna magna were increased only slightly (fig 5). Thus, the pattern of changes of the amplitude transfer function was similar to that of the amplitude of the spectral components of the CSF pulse.

\section{(4) Changes in transtentorial conduction of the CSF} pulse wave

Changes of conduction of the CSF pulse wave from the supratentorial to the infratentorial compartments were associated with the development of a transtentorial gradient of CSF pressure.

Transtentorial conduction of each spectral component of the CSF pulse was plotted against the trastentorial pressure gradient (fig 6). Values of transtentorial conduction of FW, HW2, HW3 and HW4 components of the CSF pulse wave while there was no transtentorial pressure gradient were $78.5 \pm 15 \cdot 8$, $89.4 \pm 12.1,89.0 \pm 13.7$ and $88.7 \pm 20.1 \%$, respectively. The average value of the transtentorial pressure gradient was $28.3 \mathrm{~mm} \mathrm{Hg}$ when the ipsilateral pupil was dilated. The correlation coefficient and regression line were therefore calculated in two pressure ranges (lower than $28.3 \mathrm{~mm} \mathrm{Hg}$ and over $28.3 \mathrm{~mm} \mathrm{Hg}$ ), to determine the effect of the full development of the tentorial herniation. Transtentorial conduction of the CSF pulse wave was progressively decreased during the development of the transtentorial pressure gradient while it was lower than $28.3 \mathrm{~mm} \mathrm{Hg}$. Once the transtentorial pressure gradient exceeded $28.3 \mathrm{~mm} \mathrm{Hg}$ and therefore tentorial herniation was established, the conduction of the FW and HW3 components were no longer decreased significantly, and conduction of other components was decreased at a lower rate.

Correlation between the common logarithm (base 10) of the transtentorial conduction of spectral components of the CSF pulse and the transtentorial pressure gradient was calculated because an exponential relationship was suspected (fig 6). This revealed a significant correlation for all components; the regression equations were:

$\log _{10}$ Cond $(\mathrm{FW})=-9.40 \times 10^{-3} \times \Delta \mathrm{P}_{\mathrm{CSF}}+1.64$ $(\mathrm{r}=-0.73, \mathrm{P}<0.001)$

$\log _{10}$ Cond $(H W 2)=-8.77 \times 10^{-3} \times \Delta P_{C S F}+1.82$ $(\mathrm{r}=-0.70, \mathrm{P}<0.001)$ 


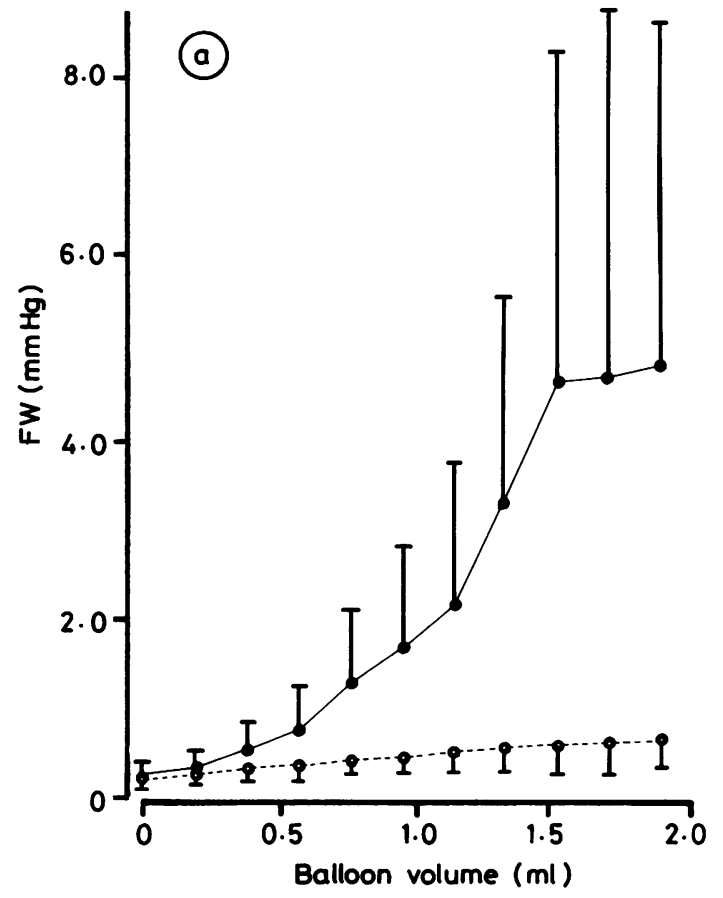

(b)
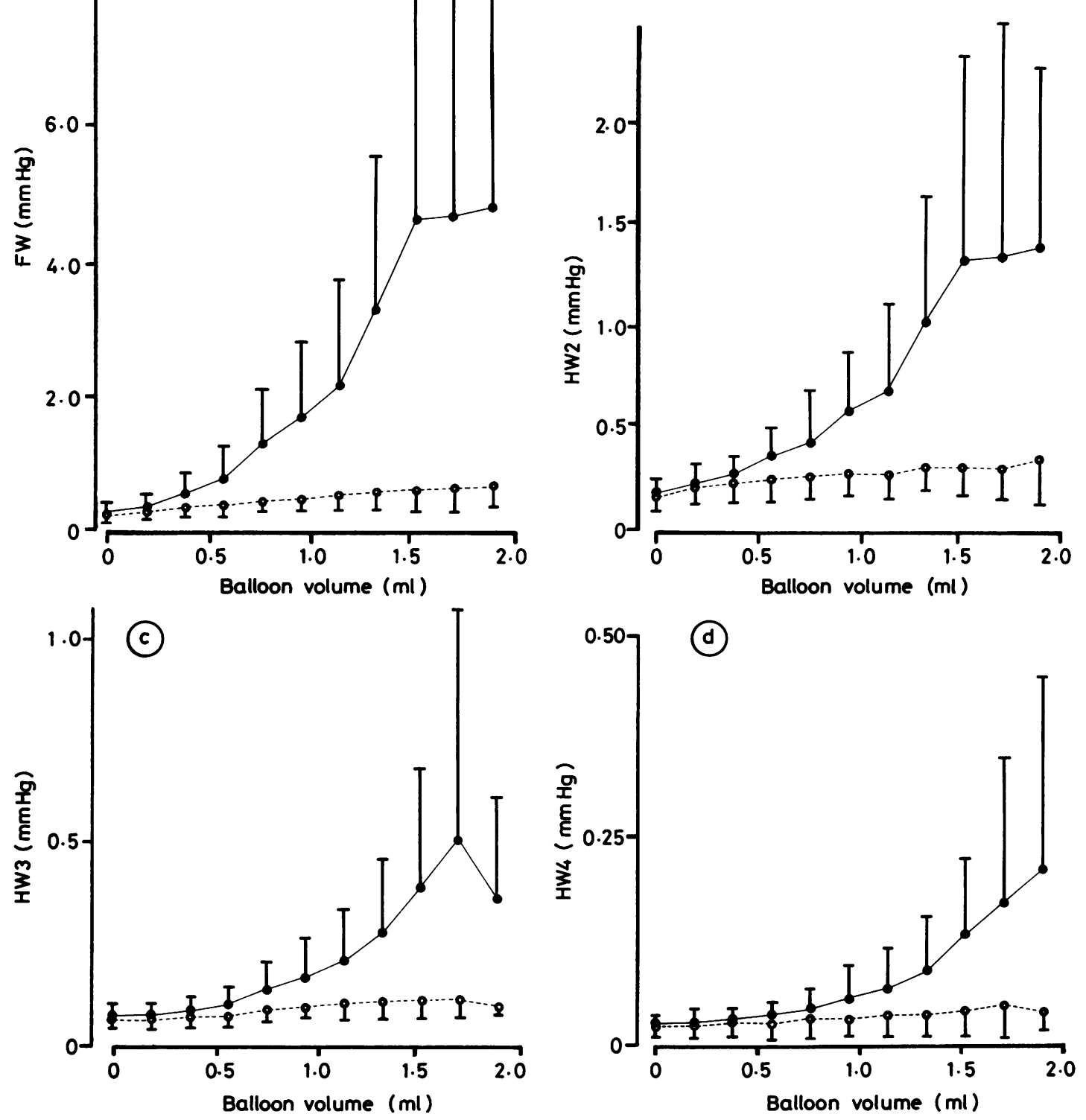

Fig 4 Changes in the amplitude of spectral components of the CSF pulse estimated in the lateral ventricle (closed circles) and cisterna magna (open circles) versus the volume in the epidural balloon. (a) FW, (b) HW2, (c) HW3 and (d) HW4 components. Mean and SD are shown.

$\log _{10}$ Cond $(\mathrm{HW} 3)=-8.05 \times 10^{-3} \times \Delta \mathrm{P}_{\mathrm{CSF}}+1.82$ $(\mathrm{r}=-0.72, \mathrm{P}<0.001)$

$\log _{10}$ Cond $(\mathrm{HW} 4)=-9.20 \times 10^{-3} \times \Delta \mathrm{P}_{\mathrm{CSF}}+1.85$ $(\mathrm{r}=-0.72, \mathrm{P}<0.001)$ where $\log _{10}$ Cond $(x)=$ common logarithm of the transtentorial conduction $(\%)$ of spectral component $(x)$ of the CSF pulse wave $(x=$ FW, HW2, HW3 or HW4)

$\Delta \mathrm{P}_{\mathrm{CSF}}=$ transtentorial pressure gradient $(\mathrm{mmHg})$ 


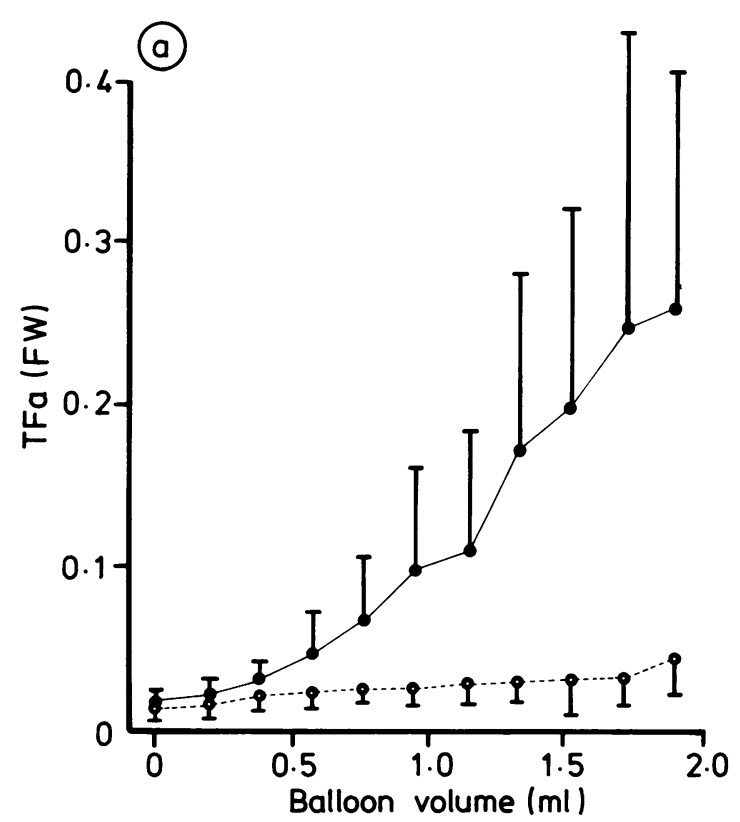

(b)
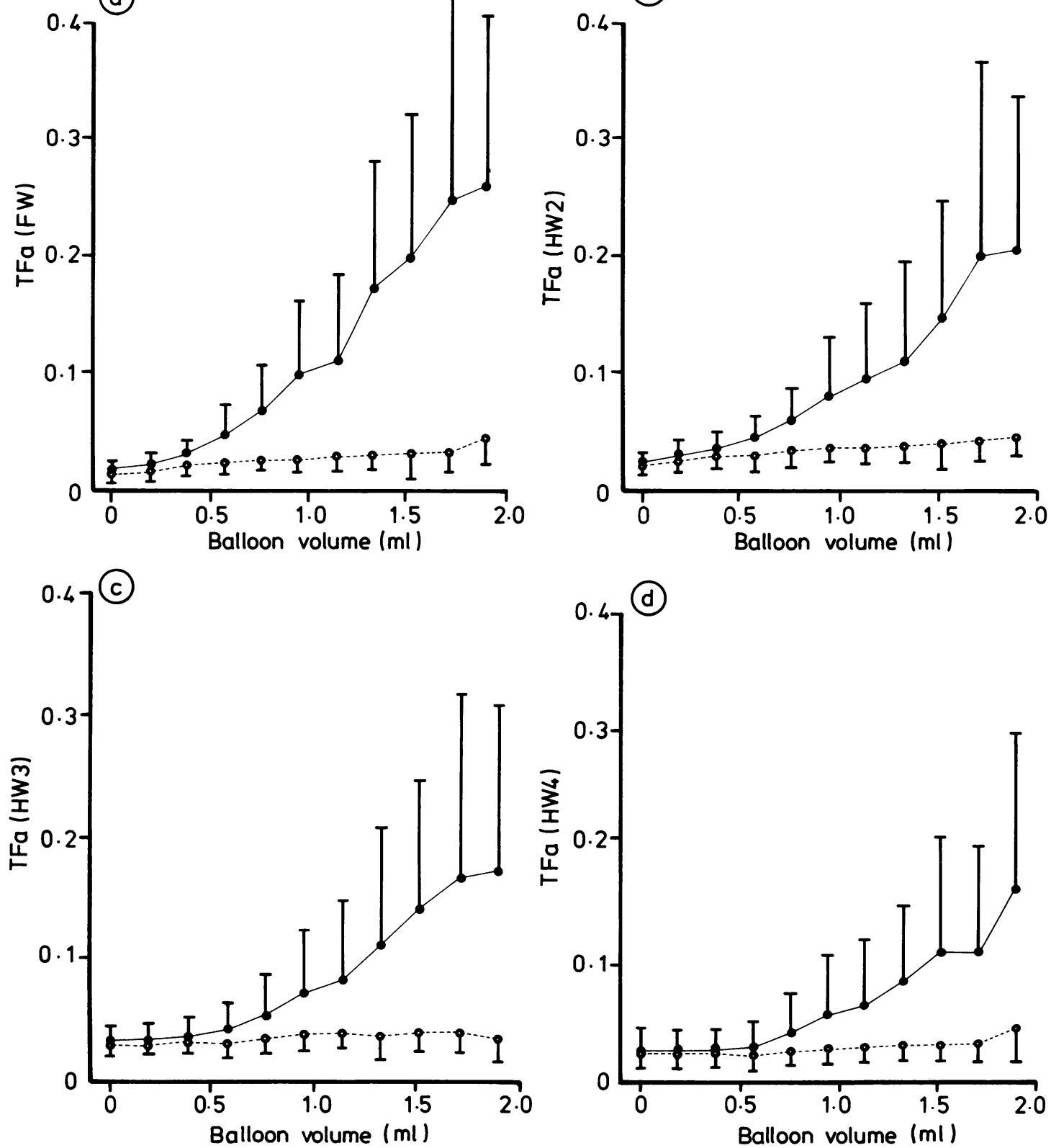

Fig 5 Changes in the amplitude transfer function (TFa $(x)$ ) of spectral components estimated in the lateral ventricle (closed circles) and cisterna magna (open circles) versus the volume in the epidural balloon. (a) $F W$, (b) $H W 2$, (c) $H W 3$ and (d) HW4 components. Mean and SD are shown. 

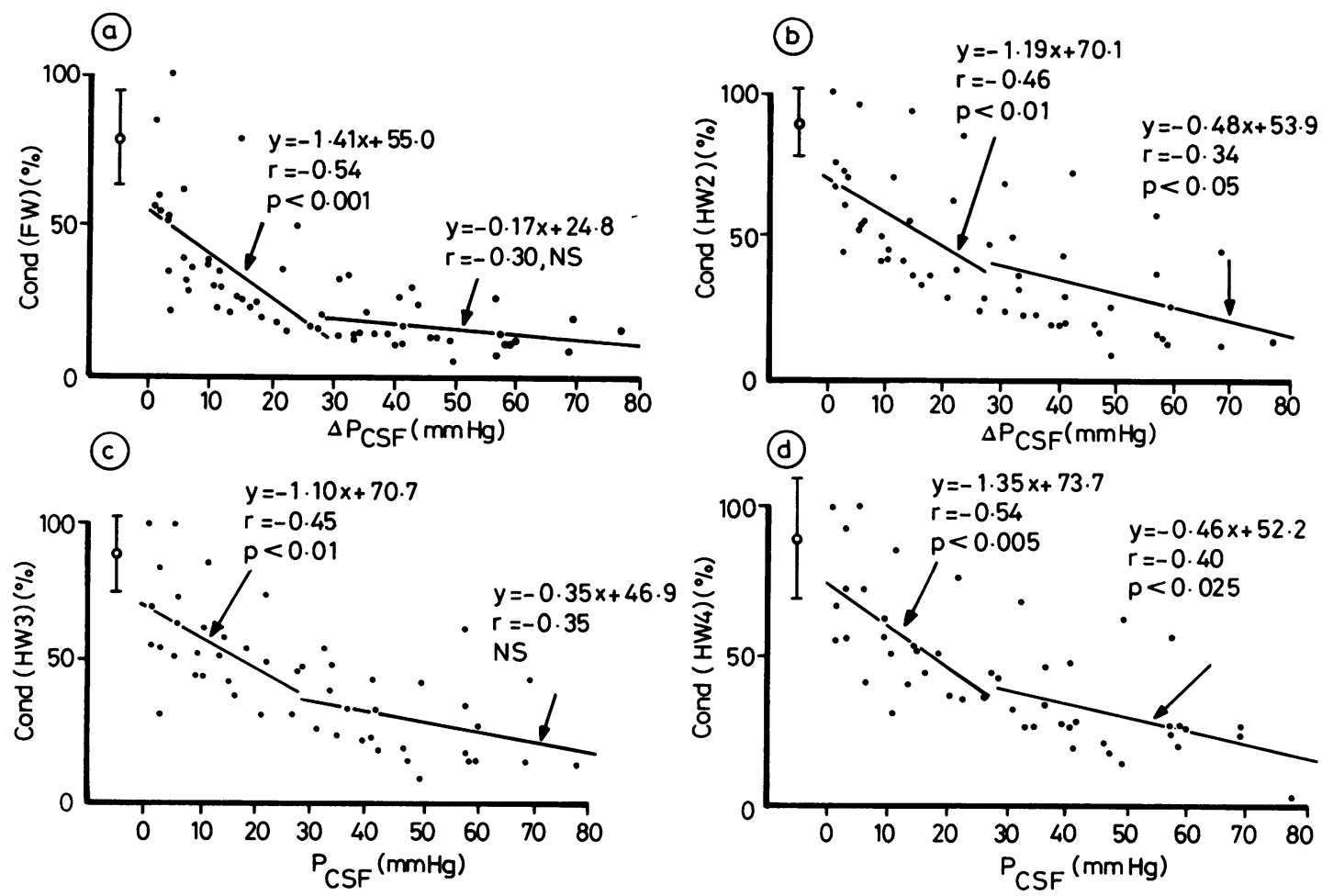

Fig 6 Changes in the transtentorial conduction (Cond $(x))$ of the CSF pulse components against the development of transtentorial pressure gradient $\left(\triangle P_{\mathrm{CSF}}\right)$. (a) FW, (b) HW2, (c) HW3 and (d) HW4 components. ${ }^{*}:$ Mean $\pm S D$ of transtentorial conduction when the pressure gradient was nil). $N S=$ not significant.

\section{Discussion}

This study revealed that the amplitude of each spectral component of the CSF pulse wave measured from the lateral ventricle increased progressively with advancing epidural brain compression. These findings are similar to the results of CSF pulse amplitude analysis using conventional methods as reported by Avezaat. ${ }^{6}$ Increasing amplitude of the spectral components of the CSF pulse wave appears to result from increasing transmission of pulsation from arterial pressure to CSF as represented by the changes of amplitude transfer function. The waveform of the CSF pulse is regulated by two main mechanisms: (a) the cerebro-vascular resistance and (b) the intracranial elastance. ${ }^{1-7}$ In other words, these two mechanisms control the efficiency of pulse transmission from arterial pressure to CSF.

In previous studies we have shown that with expansion of an extradural balloon there is a decrease in cerebrovascular resistance in the supratentorial compartment, at least up to the point where pupillary dilatation occurs. ${ }^{8}$ We have also demonstrated an increase in elastance in the supratentorial compartment with this model of increased intracranial pressure. ${ }^{9}$ In this experimental model, therefore, the condition will favour an increase in the mean level of pressure in supratentorial compartment and in the magnitude of the pulse pressure so that the CSF pressure waveform comes more and more to resemble the arterial pressure waveform.

The intracranial mass lesion pressure-volume curve has an exponential or exponential-like shape. ${ }^{10-15}$ Variation in the slope of the curve indicates changing capacity of the intracranial system for spatial compensation. This study revealed that the amplitude of spectral components of the CSF pulse wave and the amplitude transfer function from blood pressure to the CSF pulse in the supratentorial compartment also have an exponential-like relationship against the volume of an expanding epidural balloon. These findings suggest the possibility of spectral analysis of the CSF pulse as a method of estimating the intracranial elastance as proposed by Szewczykowski ${ }^{5}$ and Avezaat, ${ }^{6}$ although it would require further investigation.

A transtentorial pressure gradient appeared when 
epidural compression of brain exceeded a critical level and, thereafter, it developed progressively. The present results were similar to those reported by Fitch, ${ }^{1617}$ Langfitt $^{18}$ and Avezaat. ${ }^{6}$ The first appearance of a pressure gradient indicates the initiation of partial tentorial herniation of brain. Ipsilateral dilatation of pupil appears to indicate that the tentorial herniation is complete. Fitch ${ }^{17}$ reported that the average value of transtentorial pressure gradient, when unilateral pupillary dilatation occurred, was between 21 and $34 \mathrm{~mm} \mathrm{Hg}$ in experiments using dogs. The corresponding transtentorial pressure gradient at which unilateral pupillary dilatation appeared was $28.3 \mathrm{~mm} \mathrm{Hg}$ in these studies in the cat. These findings suggest that the degree of transtentorial pressure gradient may be more important for the development of tentorial herniation of brain than the absolute value of supratentorial intracranial pressure.

The amplitudes of all four spectral components of the CSF pulse wave were similar in both the supraand infratentorial compartments at the onset of epidural brain compression, confirming that the CSF pulse wave is freely conducted through the CSF space when there is no block. As reported by several authors, ${ }^{615-18}$ supratentorial pressure is not transmitted uniformly to the infratentorial compartment when epidural compression is advanced, and causes a transtentorial pressure gradient. This was also the case for conduction of the various components of the CSF pulse wave from the supratentorial to infratentorial compartments. Transtentorial conduction of all four spectral components of the CSF pulse was decreased rapidly in the early stage of development of transtentorial pressure gradient. In contrast, any further decrement of the CSF pulse conduction was very small after tentorial herniation was established. Decreasing conduction of the CSF pulse indicates progressive block of the CSF pathway at the tentorial hiatus. Significant correlation between the common logarithm of the transtentorial conduction of spectral components of the CSF pulse wave and the transtentorial pressure gradient suggests that blockage of the tentorial hiatus develops in an exponential fashion against the pressure gradient. These findings raise the possibility that monitoring of CSF pulse conduction could serve as an early indicator of tentorial blockage, that is tentorial herniation, which is more sensitive that the monitoring of an established transtentorial pressure gradient.

This work was supported by Action Research grant number $\mathrm{A} / 8 / 1346$.

\section{References}

1 Chopp M, Portnoy HD. System analysis of intracranial pressure. Comparison of volume-pressure test and
CSF-pulse amplitude analysis. J Neurosurg 1980;53: 516-27.

2 Hirai $\mathrm{O}$, Handa $\mathrm{H}$, Ishikawa $\mathrm{M}$. Intracranial pressure pulse waveform: Considerations about its origin and methods to estimating intracranial pressure dynamics. Brain Nerve (Tokyo) 1982;34:1059-65.

3 Nitta M. The effect of increased intracranial pressure on pulse wave. Neurol Med Chir Tokyo 1982;22:193-200.

4 Portnoy HD, Chopp M, Branch C, Shannon MB. Cerebrospinal fluid waveform as an indicator of cerebral autoregulation. Neurosurg 1982;56:666-78.

5 Szewczykowski J, Sliwka S, Kunicki A, Dytko P, Korsak-Sliwka J. A fast method of estimating the elastance of the intracranial system. A practical application in neurosurgery. $J$ Neurosurg 1977;47:19-26.

6 Avezaat CJJ, van Eijndhoven JHM. Cerebrospinal Fluid Pulse Pressure and Craniospinal Dynamics. A Theoretical, Clinical and Experimental Study. Hague, Netherland, A Jongbloed en Zoon, 1984:101-30.

7 Hamer J, Alberti E, Hoyer S, Wiedemann K. Influence of systemic and cerebral vascular factors on the cerebrospinal fluid pulse waves. $J$ Neurosurg 1977;46:36-45.

8 Miller JD, Stanek AE, Langfitt TW. Cerebral blood flow regulation during experimental brain compression. J Neurosurg 1973;39:186-96.

9 Leech P, Miller JD. Intracranial volume-pressure relationships during experimental brain compression in primates. J Neurol Neurosurg Psychiatry 1974; 37:1093-8.

10 Löfgren J, von Essen C, Zwetnow NN. The pressurevolume curve of the cerebrospinal fluid space in dogs. Acta Neurol Scand 1973;49:557-74.

11 Shulman K, Marmarou A. Pressure-volume considerations in infantile hydrocephalus. Dev Med Child Neurol 1971;13 (Supp 25):90-5.

12 Miller JD, Garibi J, Pickard JD: Induced changes of cerebrospinal fluid volume. Effects during continuous monitoring of ventricular fluid pressure. Arch Neurol 1973;28:265-9.

13 Miller JD, Sullivan HG, Becker DP. CSF pressurevolume studies and hydrocephalus. $J$ Neurosurg Sci 1978;22:147-59.

14 Sullivan HG, Miller JD, Becker DP, Flora RE, Allen GA. The physiological basis of intracranial pressure change with progressive epidural brain compression. An experimental evaluation in cats. $J$ Neurosurg 1977; 47:532-50.

15 Langfitt TW. Increased intracranial pressure. Clin Neurosurg 1969;16:436-71.

16 Fitch W., McDowall DG. Effect of halothane on intracranial pressure gradients in the presence of intracranial space-occupying lesions. $\mathrm{Br} J$ Anaesth 1971;43:904-12.

17 Fitch W, McDowall DG. Systemic vascular responses to increased intracranial pressure. 1. Effects of progressive epidural balloon expansion on intracranial pressure and systemic circulation. $J$ Neurol Neurosurg Psychiatry 1977;40:833-42.

18 Langfitt TW, Weinstein JD, Kassell NF, Simeone FA. Transmission of increased intracranial pressure. 1. Within the craniospinal axis. J Neurosurg 1964; 21:989-97. 